\title{
FPR2 serves a role in recurrent spontaneous abortion by regulating trophoblast function via the PI3K/AKT signaling pathway
}

\author{
ANNA LI ${ }^{1 *}$, SHUXIAN LI ${ }^{1 *}$, CHONGYU ZHANG ${ }^{2 *}$, ZHENYA FANG $^{1}$, \\ YAQIONG SUN ${ }^{1}$, YANJIE PENG ${ }^{1}$, XIETONG WANG ${ }^{1,3}$ and MEIHUA ZHANG ${ }^{1}$
}

${ }^{1}$ Key Laboratory of Birth Regulation and Control Technology of National Health Commission of China, Maternal and Child Health Care Hospital of Shandong Province, Jinan, Shandong 250014; ${ }^{2}$ Department of Chronic Disease, Center for Disease Control and Prevention of Wulian, Rizhao, Shandong 262300; ${ }^{3}$ Department of Obstetrics and

Gynecology, Provincial Hospital Affiliated to Shandong University, Jinan, Shandong 250021, P.R. China

Received June 29, 2021; Accepted September 10, 2021

DOI: $10.3892 / \mathrm{mmr} .2021 .12478$

\begin{abstract}
Recurrent spontaneous abortion (RSA) effects both the physical and mental health of women of reproductive age. Trophoblast dysfunction may result in RSA due to shallow placental implantation. The mechanisms underlying formyl peptide receptor 2 (FPR2) on the biological functions of trophoblasts remain to be elucidated. The present study aimed to explore the potential functions of FPR2, a $G$ protein-coupled receptor, in placental trophoblasts. The location and expression levels of FPR2 in the villi tissue of patients with RSA were detected using immunohistochemical staining, reverse transcription-quantitative PCR and western blotting. Following the transfection of small interfering RNA targeting FPR2 in HTR-8/SVneo cells, a Cell Counting Kit-8 assay was used to determine the levels of cell viability. Flow cytometry was used to examine the levels of cell apoptosis and gap closure and Transwell assays were carried out to evaluate the levels of cell migration and invasion. A tube formation assay was performed to detect the levels of capillary-like structure formation. Western blotting was used to detect the expression levels of proteins in the associated signaling pathways. The expression of FPR2 was present in villi trophoblasts and was markedly increased in patients with RSA. The levels
\end{abstract}

Correspondence to: Dr Xietong Wang or Dr Meihua Zhang, Key Laboratory of Birth Regulation and Control Technology of National Health Commission of China, Maternal and Child Health Care Hospital of Shandong Province, 238 Jingshi East Road, Jinan, Shandong 250014, P.R. China

E-mail: xietong789656@163.com

E-mail: meihua2013@163.com

*Contributed equally

Key words: recurrent spontaneous abortion, formyl peptide receptor 2, trophoblasts, $\mathrm{PI} 3 \mathrm{~K}, \mathrm{AKT}$ of trophoblast invasion, migration and tube formation were markedly increased following FPR2 knockdown, whereas the levels of apoptosis were markedly decreased. In addition, FPR2 knockdown caused an increase in the phosphorylation levels of AKT and PI3K. Thus, FPR2 may be involved in the regulation of trophoblast function via the PI3K/AKT signaling pathway. The results of the present study provided a theoretical basis for the use of FPR2 as a target for the treatment of trophoblast-associated diseases, such as RSA.

\section{Introduction}

Spontaneous abortion refers to the termination of pregnancy at $<20$ weeks or a fetal weight of $<500 \mathrm{~g}$. Recurrent spontaneous abortion (RSA) refers to $\geq 3$ consecutive spontaneous abortions with the same spouse and this is one of the most common refractory diseases in pregnancy $(1,2)$. The causes of RSA are complex and include genetic predispositions, female anatomy, hormones, infection and mental health $(3,4)$. The causes underlying $>50 \%$ of RSAs remain to be determined and further investigation is required in the context of clinical practice (5).

The occurrence and pathogenesis of RSA is closely associated with the abnormal biological behavior and dysfunction of trophoblasts (6). Dysfunction of extravillous trophoblasts, invasion defects and uterine spiral artery remodeling are key pathological characteristics of RSA (7). However, the mechanisms underlying the dysfunctional invasion of trophoblasts and abnormal uterine spiral artery remodeling in RSA remain to be elucidated.

Formyl peptide receptor 2 (FPR2) is involved in the occurrence and development of inflammation, tumors and glucose and lipid metabolism disorders, which remain key potential therapeutic targets for a number of diseases, such as colorectal cancer, acute lung injury, COPD and diabetic retinopathy. The results of a previous study demonstrate that FPR2 is expressed in trophoblasts (8), but the specific functions remain unclear. The results of previous studies have highlighted the role of FPR2 in regulating the biological function of trophoblasts in complications associated with pregnancy (8-11). 
FPR2 expression is associated with the activation of PI3K/AKT, MAPK, phospholipase C/protein kinase C, $\mathrm{NF}-\kappa \mathrm{B}$, Janus kinase (JAK)/STAT and other signaling pathways that regulate the proliferation, migration and apoptosis of tumor cells (12-14). The PI3K/AKT signaling pathway is a classical signaling pathway involved in the regulation of cell function and metabolism through either direct regulation of nutrient transporters and metabolic enzymes, or the control of transcription factors that mediate the expression of key components of metabolic pathways (15). Targeting the $\mathrm{PI} 3 \mathrm{~K} / \mathrm{AKT}$ signaling pathway is an effective method to treat a number of diseases, such as types of cancer, and may act as an effective therapeutic target in the clinic (16). However, the role of FPR2 in affecting trophoblast function via the PI3K/AKT signaling pathway in RSA remains to be elucidated.

In the present study, clinical villi tissue samples were used to determine the expression levels and effects of FPR2 in women who had experienced RSA. The role of FPR2 in regulating the biological function of trophoblasts was elucidated using FPR2 knockdown in human HTR-8/SVneo trophoblasts in vitro. The associated signaling pathway was confirmed through the use of a PI3K inhibitor. The present study clarified the role and mechanisms underlying FPR2 in the regulation of trophoblast function and provided novel targets for the treatment of RSA.

\section{Materials and methods}

Patients and sample collection. Clinical samples were obtained from the Maternal and Child Health Care Hospital of Shandong Province between January 2020 and June 2021. The present study was approved by the Ethics Committee of the Maternal and Child Health Care Hospital of Shandong province. All patients signed informed consent for scientific research. Placental villi tissue samples were obtained from 10 women who were 6-10 weeks pregnant and had experienced spontaneous abortion $\geq 2$ times, 24-33 years old. Patients with genetic factors, endocrine imbalances, immune disruption, infection, genital malformation and other factors were excluded. In addition, 10 age-matched pregnant women voluntarily admitted to hospital for induced abortion due to unwanted pregnancy at 6-10 weeks of gestation were selected as the control (CT) samples. These healthy CTs had no history of hypertension, diabetes or adverse pregnancy. Samples were collected immediately after caesarean and washed with PBS. Half of the sample was stored in liquid nitrogen and the other half were fixed in $4 \%$ paraformaldehyde at room temperature overnight, embedded in paraffin and sectioned at $4 \mu \mathrm{m}$.

Immunohistochemical staining. The aforementioned sections were boiled in $0.01 \mathrm{~mol} / 1$ citrate buffer $(\mathrm{pH}$ 6.0) for $10 \mathrm{~min}$ for antigen retrieval and subsequently cooled to room temperature. Methanol containing $3 \% \mathrm{H}_{2} \mathrm{O}_{2}$ was used to eliminate endogenous peroxidases. After being blocked with 5\% BSA (Beijing Solarbio Science \& Technology Co., Ltd.) for $30 \mathrm{~min}$, the sections were incubated with the anti-FPR2 primary antibody (cat. no. NLS1878; Novus Biologicals, LLC; 1:500) in a wet box overnight at $4^{\circ} \mathrm{C}$. Following primary incubation, the sections were incubated with the rabbit $\operatorname{IgG}(\mathrm{H}+\mathrm{L})$ secondary antibody (cat. no. 31460 ; Invitrogen; $1: 500$ ) at $37^{\circ} \mathrm{C}$ for $1 \mathrm{~h}$. Subsequently, a diaminobenzidine reagent kit $(1: 1,000$; Sigma-Aldrich) was used. For cell culture slides, a secondary antibody conjugated to FITC (cat. no. AP132F; Sigma-Aldrich; Merck KGaA; 1:1,000) was used at $37^{\circ} \mathrm{C}$ for $1 \mathrm{~h}$.

$R N A$ extraction and reverse transcription-quantitative (RT-q) $P C R$. Total RNA was extracted from tissues using TRIzol ${ }^{\circledR}$ reagent (Thermo Fisher Scientific, Inc.) according to the manufacturer's protocol. The quality and concentration of RNA were confirmed using a NanoDrop1000 (Thermo Fisher Scientific, Inc.). Total RNA was reverse transcribed into cDNA using High-Capacity cDNA Reverse Transcription kit (cat. no. 4368814; Applied Biosystems; Thermo Fisher Scientific, Inc.) according to the manufacturer's protocol. qPCR was subsequently performed using the LightCycler II 480 (Roche Diagnostics $\mathrm{GmbH}$ ). The following thermocycling conditions were used for the qPCR: Initial denaturation at $95^{\circ} \mathrm{C}$ for $30 \mathrm{sec}$, followed by 40 cycles at $95^{\circ} \mathrm{C}$ for $5 \mathrm{sec}$, $60^{\circ} \mathrm{C}$ for $10 \mathrm{sec}, 72^{\circ} \mathrm{C}$ for $15 \mathrm{sec}$, and melting from $65^{\circ} \mathrm{C}$ to $95^{\circ} \mathrm{C}$, every $0.11^{\circ} \mathrm{C} / 1 \mathrm{sec}$, and $40^{\circ} \mathrm{C}$ for $10 \mathrm{sec}$. The expression level of mRNA was assessed using the $2^{-\Delta \Delta \mathrm{Cq}}$ method (17). The following primer pairs were used for qPCR: FPR2 forward, 5'-ATGTCCATTGTTGCCATCTGC-3' and reverse, 5'-GACGTAAAGCATGGGGTTGAG-3'; and GAPDH forward, 5'-GCACCGTCAAGGCTGAGAAC-3' and reverse, 5'-TGGTGAAGACGCCAGTGGA-3'.

Cell culture. The human trophoblast cell line HTR8/SVneo, originating from human placental trophoblast cells, was purchased from the American Type Culture Collection. Cells were cultured in RPMI-1640 medium (Gibco; Thermo Fisher Scientific, Inc.) containing 10\% FBS (Gibco; Thermo Fisher Scientific, Inc.) at $37^{\circ} \mathrm{C}$ in a $5 \% \mathrm{CO}_{2}$ incubator. When $\sim 80 \%$ confluence was reached, cells were plated onto a six-well culture plate at $1 \times 10^{5}$ cells/well for subsequent experiments. The PI3K inhibitor-LY294002 $(10 \mu \mathrm{M})$ was purchased from MedChemExpress. As control, cells in solvent groups were treated with the solvent of LY294002 (10\% DMSO, 40\% PEG300, 5\% Tween-80 and 45\% saline).

Cell transfection. Transfection of FPR2 small interfering (si)RNA purchased from Thermo Fisher Scientific, Inc. (cat. no. AM16708) was used to knockdown the FPR2 gene in trophoblasts. siR NC \#1 was purchased from Guangzhou RiboBio Co., Ltd. (cat. no. siN0000001-1-5) as the negative control (NC). HTR8/SVneo cells were inoculated into a 6-well plate and divided into two groups: siRNA-NC and siRNA-FPR2. When $\sim 50 \%$ confluence was reached, complete medium (RPMI-1640 medium supplemented with $10 \%$ FBS) was replaced by reduced-serum medium. A total of $4 \mu \mathrm{l}$ Lipofectamine ${ }^{\circledR} 2000$ transfection reagent (Invitrogen; Thermo Fisher Scientific, Inc.) and $6 \mu$ FPR2 siRNA were mixed, incubated at room temperature for $15 \mathrm{~min}$ and subsequently added into the aforementioned reduced-serum medium. Cells were incubated at $37^{\circ} \mathrm{C}$ in $5 \% \mathrm{CO}_{2}$ for $8 \mathrm{~h}$. The medium was discarded and replaced with complete medium and cultured for a further $48 \mathrm{~h}$. Proteins were extracted and the transfection efficiency was detected using western blotting. 
Transwell assay. Transwell chambers (8- $\mu \mathrm{m}$; Corning, Inc.) were coated with $60 \mu 1$ Matrigel (1:8; BD Biosciences) at $37^{\circ} \mathrm{C}$ for $1 \mathrm{~h}$. A total of $5 \times 10^{5} \mathrm{HTR} 8 / \mathrm{SVneo}$ cells suspended in $100 \mu \mathrm{l}$ serum-free medium were seeded into the upper chamber and the lower chamber was filled with $600 \mu \mathrm{l}$ RPMI-1640 medium containing $10 \% \mathrm{FBS}$. Following incubation at $37^{\circ} \mathrm{C}$ in $5 \% \mathrm{CO}_{2}$ for $48 \mathrm{~h}$, cells that migrated to the lower surface of the chamber were fixed with $4 \%$ paraformaldehyde at room temperature and stained with $0.1 \%$ crystal violet for $20 \mathrm{~min}$ at room temperature. Images were captured using an inverted microscope (Zeiss $\mathrm{GmbH}$ ) and invasive cells were counted in at least five randomly selected fields for each chamber (magnification, x200). Untreated Transwell chambers were used to detect cell migration.

Gap closure assay. HTR-8/SVneo cells were seeded in a 6-well plate at a density of $2 \times 10^{5} /$ well and cultured for 24-36 h. When $\sim 90 \%$ confluence was reached, the wound was scratched using a culture insert (ibidi, Germany). Cell debris were removed by washing three times in PBS and the medium was replaced with FBS-free medium with other treatments for $24 \mathrm{~h}$. Cell migration was observed at 0 and $12 \mathrm{~h}$ using an inverted phase contrast microscope (Zeiss $\mathrm{GmbH}$; magnification, $\mathrm{x} 4$ ) and analyzed using a High Content Analysis system (HCS; Molecular Devices, LLC).

Cell Counting Kit-8 (CCK-8) assay. HTR-8/SVneo cells were seeded into a $96-w e l l$ plate at a density of 300-5,000 cells/well and cultured at $37^{\circ} \mathrm{C}$ in $5 \% \mathrm{CO}_{2}$ for $24 \mathrm{~h}$. Subsequently, $10 \mu \mathrm{l}$ CCK-8 solution (Elabscience Biotechnology, Inc.) was added to each well and incubated for 1-4 h. Absorbance was measured at $450 \mathrm{~nm}$ using a microplate reader (BioTek Instruments, Inc.).

Flow cytometry. Adherent HTR-8/SVneo cells were washed with PBS for three times and trypsinized. Cells were resuspended in $195 \mu \mathrm{l}$ Annexin V-FITC binding solution (BD Biosciences) at a density of $5 \times 10^{5} /$ tube. Cells were subsequently mixed with $5 \mu \mathrm{l}$ Annexin V-FITC and $10 \mu \mathrm{l}$ PI staining solution (BD Biosciences) and incubated for $20 \mathrm{~min}$ at room temperature in the dark. Cell apoptotic rates were calculated by detecting the percentage of early and late apoptotic cells using flow cytometry (DxFLEX; Beckman Coulter, Inc.) and CytExpert software (version 2.0.0.283; Beckman Coulter, Inc.).

Western blotting. Villi tissues were cut into small pieces and homogenized using Tissue Grinders (Potter-Elvehjem) on ice and lysed in RIPA buffer (Sigma-Aldrich; Merck KGaA) with $1 \mathrm{mmol} / 1 \mathrm{PMSF}$. Total protein was quantified using a BCA assay and $20 \mu \mathrm{g}$ protein of each sample was separated by 7.5\% SDS-PAGE. The separated proteins were subsequently transferred to PVDF membranes and blocked with 5\% skimmed milk at room temperature for $1 \mathrm{~h}$. The membranes were incubated with specific primary antibodies, including anti-FPR2 (cat. no. 63023, Abcam, 1:1,000), anti-phosphorylated (p)-AKT (cat. no. 4060; Cell Signaling Technology, Inc.; 1:1,000), anti-AKT (cat. no. 9272; Cell Signaling Technology, Inc.; 1:1,000), anti-PI3K (cat. no. 4292; Cell Signaling Technology, Inc.; 1:1,000), anti-p-PI3K (cat. no. 4228; Cell Signaling Technology, Inc.; 1:1,000) and anti- $\beta$-actin (cat. no. 66009; 1:2,000; ProteinTech Group, Inc.) overnight at $4^{\circ} \mathrm{C}$. Following primary incubation, membranes were incubated with HRP-conjugated secondary antibody (cat. no. 15015; 1:5,000; ProteinTech Group, Inc.) at room temperature for $1 \mathrm{~h}$. The blots were developed using the ECL Detection kit (Thermo Fisher Scientific, Inc.). Protein bands were semi-quantified using the Amersham Imager 600 (Cytiva) and analyzed using ImageJ 1.51 software (National Institutes of Health).

Statistical analysis. All experiments were repeated at least three times. Data were collected, analyzed and visualized using GraphPad Prism version 8.0 (GraphPad Software, Inc.). The continuous variables with normal distribution are presented as the mean \pm SD. Differences between the si-NC group and si-FPR2 groups for migration, proliferation, invasion, apoptosis, tube formation rate and the phosphorylation of PI3K and AKT were analyzed using unpaired Student's t-tests. Comparisons between three or more groups were conducted using one way analysis of variance (ANOVA) and the P-values were adjusted using Bonferroni post hoc test. $\mathrm{P}<0.05$ was considered to indicate a statistically significant difference.

\section{Results}

Expression of FPR2 significantly increases in placenta tissues from patients with RSA. To confirm the association between FPR2 and RSA, the location and expression of FPR2 in the placenta of women with RSA was investigated. As demonstrated in Fig. 1A, results of the immunohistochemical analysis revealed that FPR2 was located on the cytotrophoblasts of villi in the placenta tissue. In addition, the expression levels of FPR2 in the villi of patients with RSA were significantly increased, compared with the CT group. In order to verify the changes in expression levels of FPR2 in the villi of patients with RSA, western blotting and RT-qPCR were performed using villi tissues derived from 10 patients in each of the RSA and CT groups. Consistent with the results of the immunohistochemical staining, the protein expression levels of FPR2 in the villi of patients with RSA were increased compared with the CT group (Fig. 1B). The mRNA expression levels of FPR2 in women with RSA was significantly increased compared with the CT group (Fig. 1C). These results suggested that an increase in the levels of FPR2 is associated with RSA.

FPR2 knockdown inhibits apoptosis in trophoblasts. As the biological dysfunction of trophoblasts is closely associated with the pathogenesis of RSA (18), the impact of FPR2 knockdown on the biological function of trophoblasts was investigated. As demonstrated in Fig. 2A, FPR2 was expressed in HTR8/SVneo cells and predominantly located in the cytoplasm and cell membrane. Following the knockdown of FPR2 using siRNA, the transfection efficiency was verified using western blotting. Compared with si-NC cells, the protein expression levels of FPR2 were significantly decreased in si-FPR2 cells (Fig. 2B). CCK-8 assays demonstrated that FPR2 knockdown exhibited no significant effect on cell proliferation, compared with si-NC cells (Fig. 2C). In addition, the knockdown of FPR2 significantly attenuated the apoptosis of trophoblasts compared with the si-NC group (Fig 2D). 
A

CT

RSA

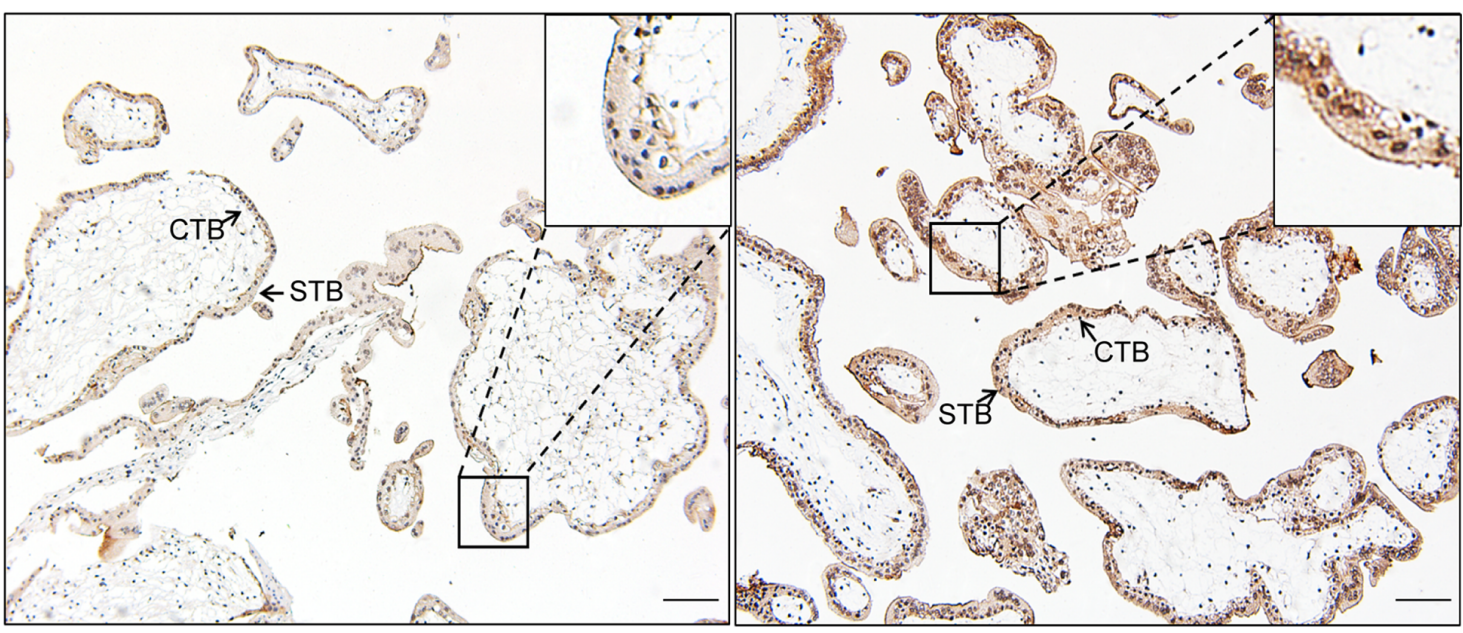

B

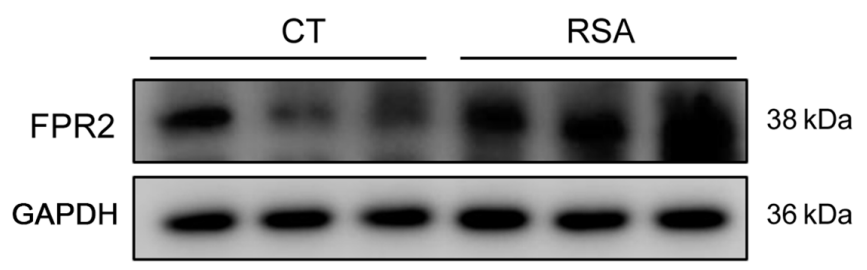

C
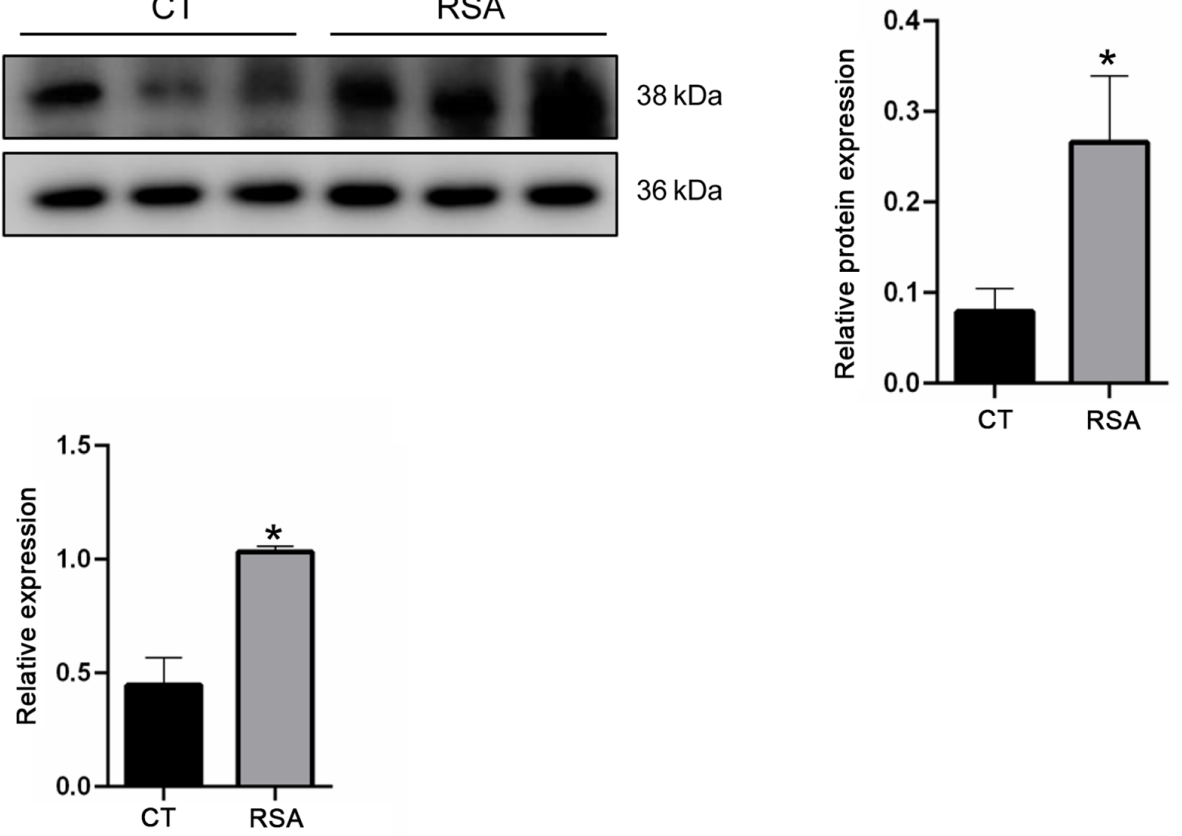

Figure 1. FPR2 is upregulated in human PE placenta tissues. (A) The expression of FPR2 in the villi of first trimester placenta of RSA and normal pregnancy women were analyzed by immunohistochemical staining. Scale bars $=100 \mu \mathrm{m}$. (B) The protein expression of FPR2 in placenta tissues was analyzed by western blotting. Protein levels were quantified by densitometry analyzing using Image J software. (C) The mRNA expression of FPR2 in the placenta was analyzed by reverse transcription-quantitative PCR. Data are presented as mean \pm SD obtained from at least three independent experiments. ${ }^{*} \mathrm{P}<0.05$. FPR2, formyl peptide receptor 2; CT, control; RSA, recurrent spontaneous abortion; CTB, cytotrophoblasts; STB, syncytiotrophoblasts.

FPR2 knockdown ameliorates migration, invasion and tube formation ability of trophoblasts. To further explore the biological significance of FPR2 in trophoblast function, the role of FPR2 in the regulation of trophoblast migration and invasion was investigated using gap closure and Transwell assays. FPR2 knockdown mitigated the migration rate of HTR8/SVneo cells compared with the si-NC group (Fig. 3A and B). In addition, results of the Transwell assay demonstrated that the levels of invasion decreased in HTR8/SVneo cells following FPR2 knockdown compared with the si-NC group (Fig. 3C). Tube formation assays demonstrated that HTR8/SVneo cells treated with si-FPR2 exhibited an increased capability of forming capillary-like structures, compared with the si-NC group (Fig. 3D).
FPR2 affects the migration and invasion of trophoblasts via the PI3K/AKT pathway. The results of a previous study revealed that activation of FPR 2 triggers the activation of PI3K in cancer cells $(19,20)$. Thus, to investigate the involvement of PI3K in the regulation of trophoblast proliferation by FPR2, western blotting was used to detect the phosphorylation levels of PI3K and AKT. The results demonstrated that FPR2 knockdown increased the activation of the PI3K signaling pathway compared with the si-NC+ Solvent group. In addition, in the presence of LY294002 (10 $\mu \mathrm{M}$; MCE), a specific PI3K inhibitor, the si-FPR2-mediated increase in $\mathrm{p}$-AKT was reversed, with no significant changes in FPR2 expression (Fig. 4A). Gap closure assays revealed no 
A

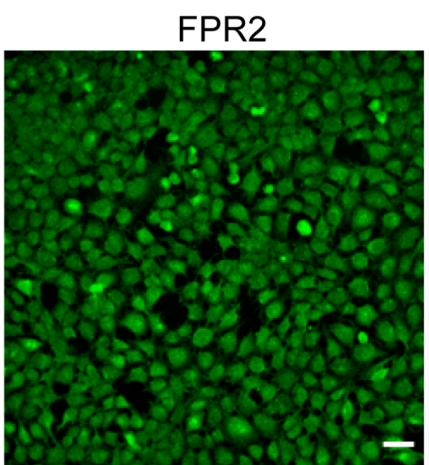

DAPI Merged

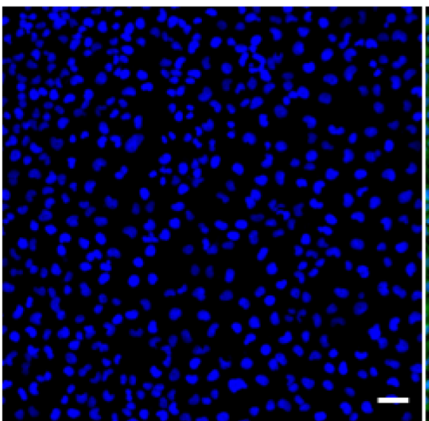

B
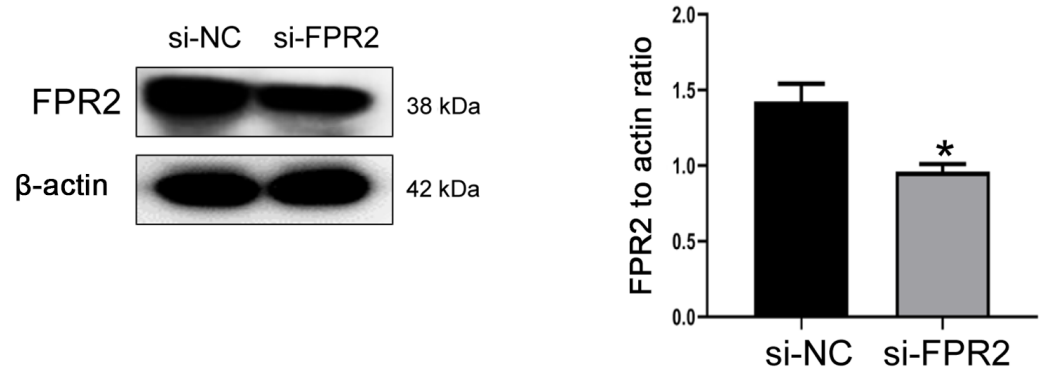

C

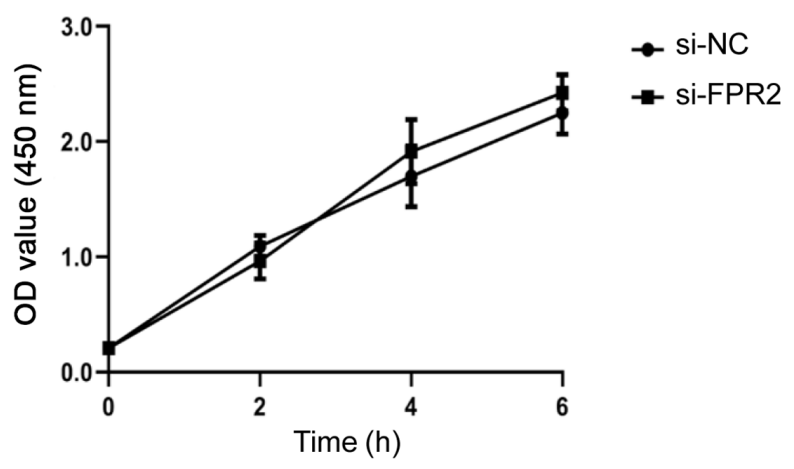

D

si-NC

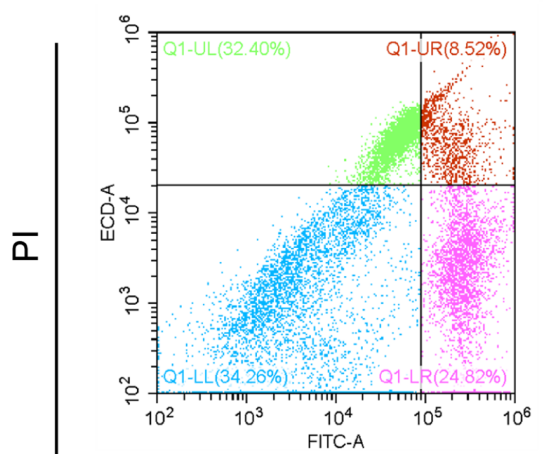

si-FPR2

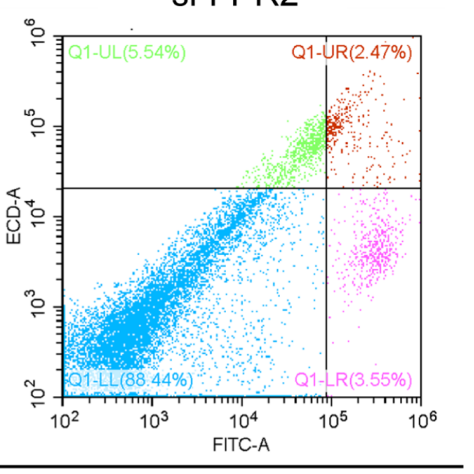

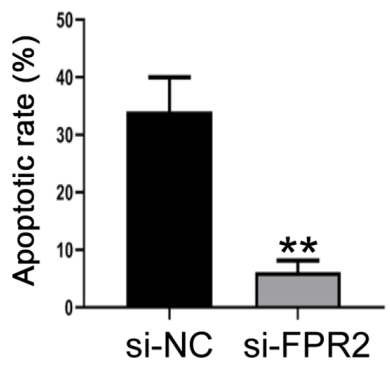

Annexin V

Figure 2. FPR2 knockdown suppresses trophoblast apoptosis. (A) Immunofluorescence of FPR2 in HTR-8/SVneo (HTR8) cells. (B) Western blotting was used to analyze the expression of FPR2 following $48 \mathrm{~h}$ si-FPR2 transfection. (C) CCK8 assay were used to evaluate proliferation in HTR8-siFPR2 cells. (D) Flow cytometry was performed to detect cell apoptosis. Representative flow cytometry data were shown. Data are presented as mean \pm SD obtained from at least three independent experiments. "P $<0.05,{ }^{* *} \mathrm{P}<0.01$. FPR2, formyl peptide receptor 2 ; si, small interfering; PI, propidium iodide; Annexin V FITC, Annexin V labeled with green fluorescent probe FITC.

significant difference in the migration of HTR8/SVneo cells following treatment with si-FPR2 and LY294002, compared with the si-NC+ Solvent group (Fig. 4B). Similarly, the si-FPR2-mediated increase in cell invasion was inhibited following treatment with LY294002 (Fig. 4C). Collectively, these findings indicated that FPR2 affects the migration and invasion of HTR8/SVneo cells via the PI3K-AKT signaling pathway. 

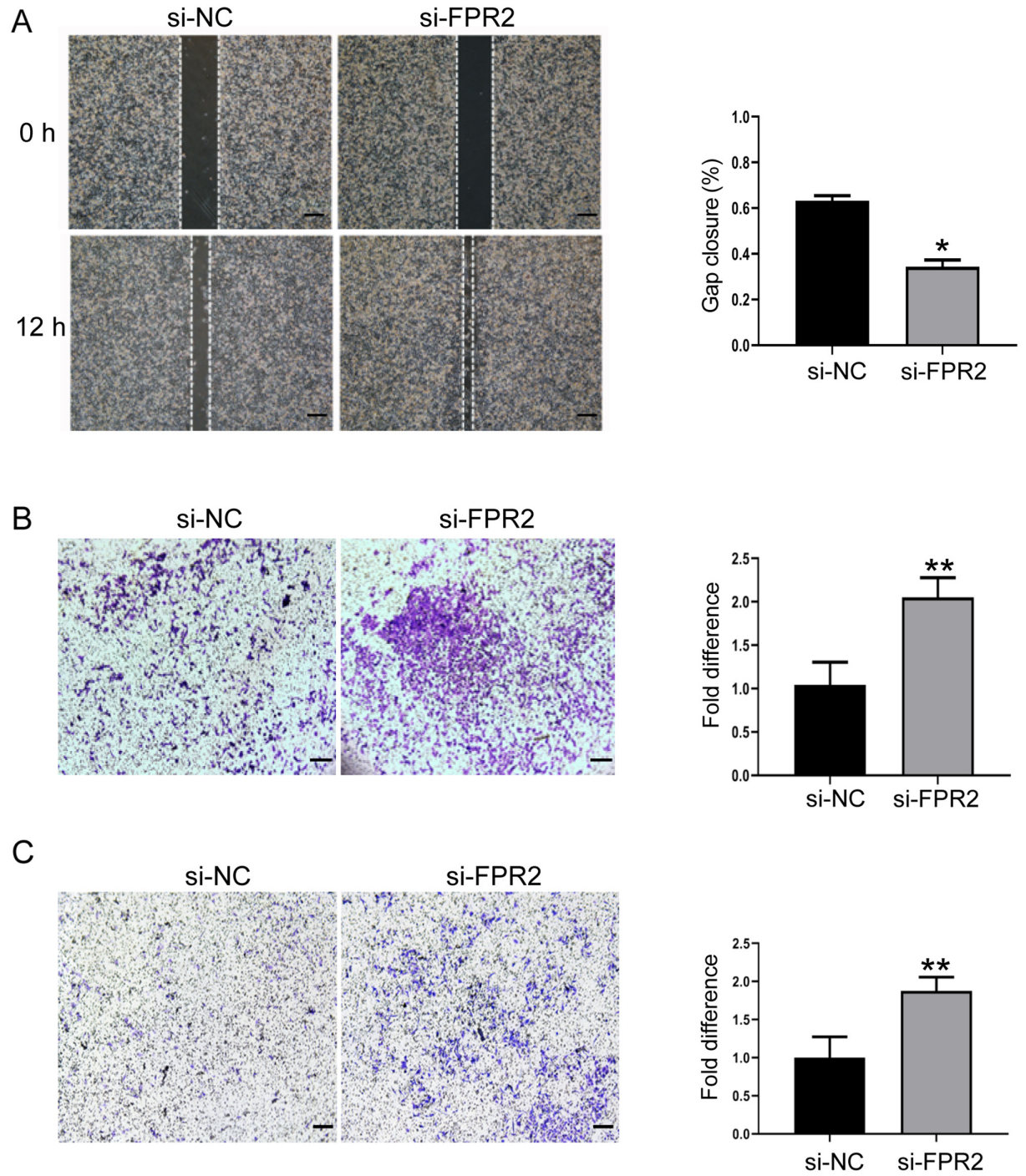

D
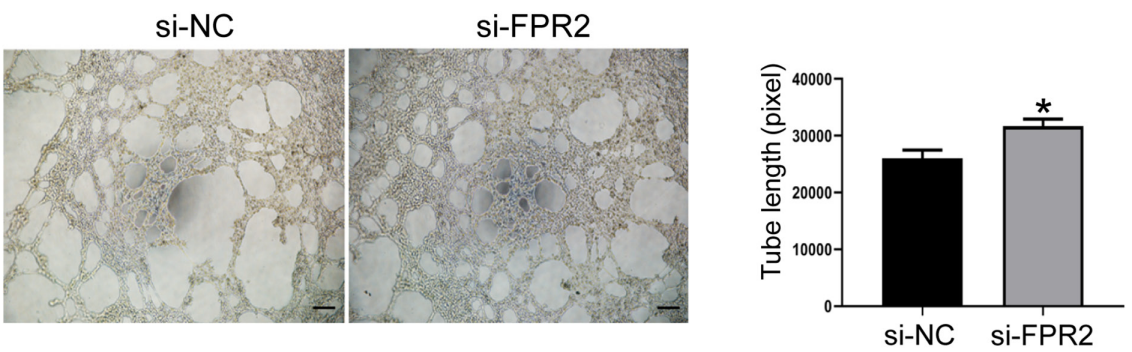

Figure 3. FPR2 deficiency ameliorates migration, invasion and tube formation ability of HTR8 cells. (A) Gap closure and (B) Transwell assays were used to estimate cell migration transfected with si-FPR2. Scale bars=200 $\mu \mathrm{m}$. (C) Transwell assays were used to assess invasion in the presence of si-FPR2, Scale bars $=200 \mu \mathrm{m}$. (D) Tube formation assay was used to examine the tube formation ability of HTR8 cells. Scale bars=200 $\mu \mathrm{m}$. Representatives of light microscopic images are shown. Data are presented as mean \pm SD obtained from at least three independent experiments. " $\mathrm{P}<0.05$, "** $\mathrm{P}<0.01$. FPR2, formyl peptide receptor 2; si, small interfering; $\mathrm{NC}$, negative control.

\section{Discussion}

The occurrence of RSA is closely associated with the abnormal biological function of trophoblasts (18). Trophoblasts are cells that constitute the maternal-fetal interface and serve a key role during pregnancy. The normal biological function of trophoblasts is important to ensure an adequate nutrient supply and the secretion of essential hormones for fetal growth and development (21). When a fertilized egg is implanted, the anchoring villi in the placenta differentiate into syncytiotrophoblasts and cytotrophoblasts. Cytotrophoblasts gradually differentiate to the infiltrating surface layer, forming extravillous trophoblasts (EVTs) (22). EVTs are divided into mesenchymal trophoblasts and intravascular trophoblasts, according to their different functions. The main function of mesenchymal trophoblasts is to infiltrate the basal layer of the uterus, a mechanism that is strictly regulated to avoid excessive invasion and implantation (23). The main role of EVTs is to invade the uterine spiral artery, participate in the remodeling of the spiral artery and ensure blood perfusion of the placenta (24). Insufficient inva- 

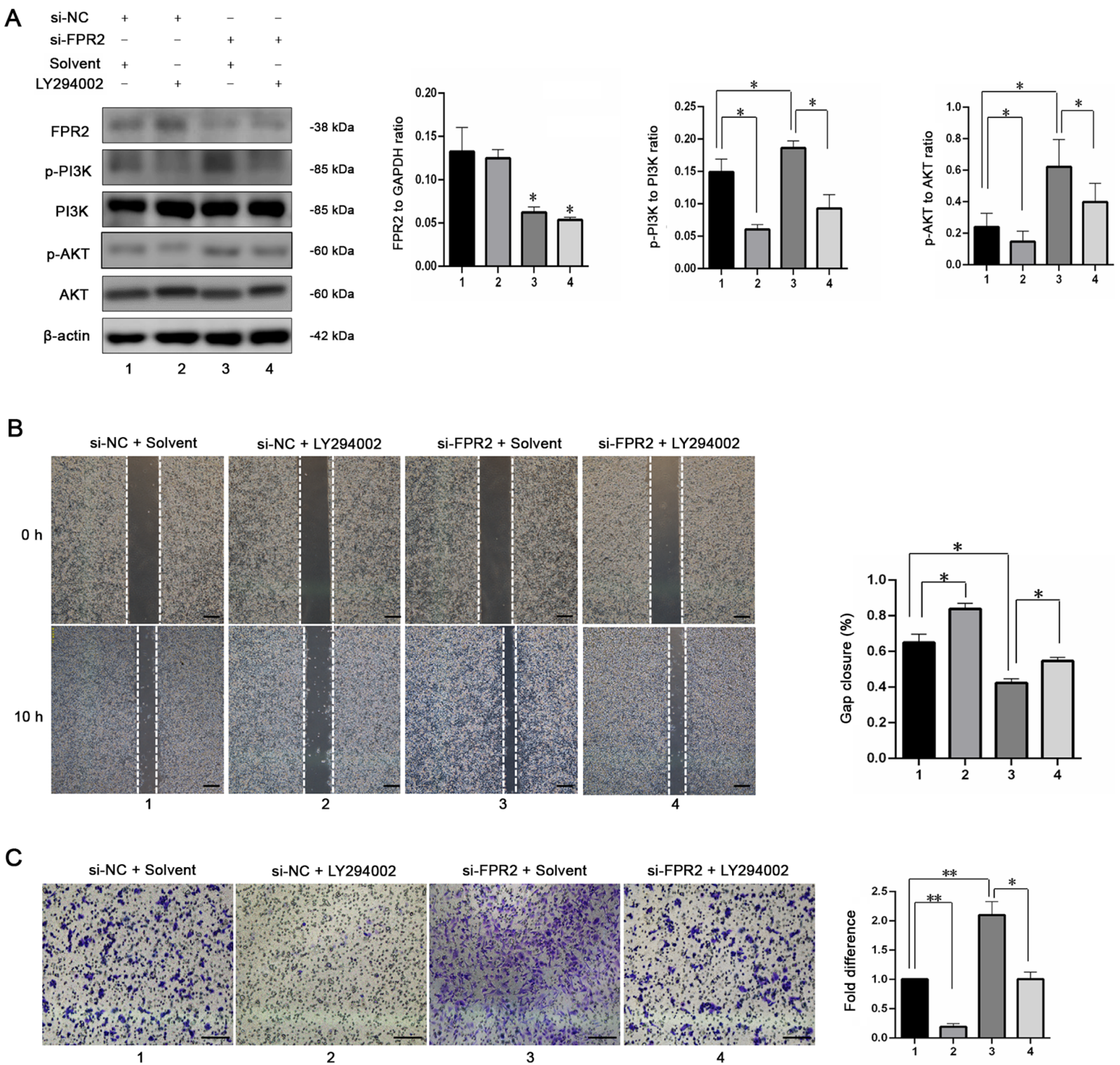

Figure 4. Knockdown of FPR2 promotes cell migration and invasion through the PI3K-AKT pathway. (A) Western blotting of FPR2, p-PI3K, PI3K, p-AKT and AKT in HTR8 cells transfected with si-FPR2 or si-NC in the presence or absence of LY294002 and densitometry quantification of western blots. (B) Gap closure and (C) Transwell assays of FPR2 silencing cells in the presence of LY294002. Scale bars $=200 \mu \mathrm{m}$. Data are presented as mean \pm SD obtained from at least three independent experiments. ${ }^{*} \mathrm{P}<0.05,{ }^{* *} \mathrm{P}<0.01$. FPR2, formyl peptide receptor 2 ; p-, phosphorylated; si, small interfering; NC, negative control.

sion of trophoblast causes spiral arteriole remodeling in the decidual layer, which is markedly shallower in uterine muscle layer compared with normal pregnancy, leading to the poor formation of the placental vascular network (25). Pathological changes associated with placental ischemia, such as hypoxia and metabolic disorders then occur, leading to pregnancy complications. However, the mechanisms underlying trophoblast dysfunction in patients with RSA remain to be elucidated.

The results of previous studies have demonstrated that dysfunctional EVTs that affect the remodeling of uterine spiral artery during the development of the placenta are a key pathological feature of pregnancy complications, such as RSA and pulmonary embolisms $(26,27)$. HTR-8/SVneo cells are generated using freshly isolated extravillous cytotrophoblasts from the first trimester placenta transfected with a plasmid containing the simian virus 40 large $\mathrm{T}$ antigen (SV40), which represents early human villous trophoblasts (28). These cells have previously been used as models of RSA for the research of diseases associated with the placenta and to observe the effect of FPR2 expression on trophoblast function (29). The results of a previous study demonstrate that HTR-8/SVneo cells contain two populations, one of epithelial and one of mesenchymal origin (30). Furthermore, the results of previous studies demonstrate that these cells act as ideal models to study a number of diseases, such as chorionic amnionitis (8) and the reproductive toxicity of silica nanoparticles (31-33).

FPR2, a member of the formyl-peptide receptor family, is a G protein-coupled receptor (34). The physiological and pathological functions of FPR2 have been extensively studied using FPR2 agonists, RNA interference technology and FPR2 gene knockout mice, combined with in vitro and in vivo experiments. FPR 2 not only regulates the chemotaxis, migration and phagocytosis of leukocytes, but also serves a role in defense 
reactions and inflammatory diseases (22).FPR2 is also involved in the occurrence of neurodegenerative diseases, tumors and disorders associated with glucose and lipid metabolism and is a potential therapeutic target for a number of diseases (35-37). In addition, abnormal expression of FPR 2 has been discovered in a number of types of tumor tissues and is involved in the proliferation and invasion of tumor cells $(38,39)$. FPR2 also affects tumor growth and metastasis by acting on the tumor microenvironment to cause the abnormal autophagy of tumor cells (40). Trophoblasts have biological characteristics similar to tumor cells and are highly invasive. However, unlike tumor cells, trophoblasts are controlled by a number of regulatory factors that are space- and time-dependent and do not have the potential for infinite proliferation and differentiation (41). The results of our previous study demonstrated that human placental trophoblasts express FPR2 (8), but the mechanism underlying FPR2 in regulating the biological function of trophoblasts remains to be elucidated and its role in pregnancy requires further investigation.

In the present study, FPR2 was expressed in the trophoblasts of villi in early pregnancy and the expression levels were significantly increased in patients with RSA, suggesting that the abnormal expression of FPR2 in trophoblasts may be associated with the pathogenesis of RSA. In addition, the levels of trophoblast migration, invasion and proliferation were markedly increased, while the levels of apoptosis were reduced following FPR2 knockdown in HTR8/SVneo cells. This highlighted the potential role of FPR2 in the regulation of trophoblast function. Collectively, these results indicated that FPR2 may be involved in the occurrence of RSA by regulating the biological function of trophoblasts.

The signaling pathways involved in regulating the biological function of trophoblasts include tyrosine-protein kinase JAK1/STAT, PI3K/AKT, MAPK and TGF- $\beta$ (42). The results of a previous study demonstrate that activation of the $\mathrm{NF \kappa B}$ signaling pathway is also involved in the regulation of trophoblast cell function (43). The expression of FPR2 is associated with the activation of PI3K/AKT, MAPK, NF- $\mathrm{BB}$ and other signaling pathways, which also serve key roles in the regulation of tumor cells, including cell proliferation, migration and apoptosis. Kyoto Encyclopedia of Genes and Genomes functional enrichment analysis reveals an association between FPR2 and the PI3K/AKT signaling pathway (12). The association between FPR2 and the $\mathrm{PI}$ KK/AKT signaling pathway requires further investigation in trophoblasts.

The results of the present study demonstrated that FPR2 knockdown caused the phosphorylation of AKT and PI3K. AKT is the direct target protein of the PI3K signaling pathway and is associated with cell proliferation, differentiation, apoptosis and migration (44-46). Functional analyses in the present study revealed that FPR 2 may be involved in the regulation of trophoblast function through the PI3K/AKT signaling pathway. Furthermore, use of a PI3K/AKT signaling pathway agonist or an FPR2 inhibitor in the early pregnancy of patients with RSA may improve the function of trophoblasts in the placenta (47) and may provide novel therapeutic targets for the treatment of diseases associated with the placenta. However, increasing the activation of the PI3K/AKT signaling pathway may cause adverse side effects, such as excessive cell proliferation and cancer development (48). Thus, further investigations into the specific dosages are required. In addition, the PI3K/AKT signaling pathway mediates cellular metabolism through a number of key downstream substrates, such as tuberin, protein kinase gsk3 and the FOXO transcription factors (15). Thus, the effects of downstream substrates of the PI3K/AKT signaling pathway on the functions of FPR2 in trophoblasts require further investigation.

The present study had a number of limitations, such as a lack of in vivo investigations. Thus, future investigations should use a placenta-specific FPR2 knockout mouse model to study the FPR2-associated dysfunction of trophoblasts in vivo. An in vivo model is essential to further elucidate the pathogenic mechanisms underlying RSA.

In conclusion, FPR2 serves a key role in the regulation of trophoblasts through the PI3K/AKT signaling pathway and may be involved in RSA development. Further elucidating the mechanisms underlying FPR2 in cell proliferation, migration, invasion and apoptosis may uncover novel disease markers and therapeutic targets in RSA.

\section{Acknowledgements}

Not applicable.

\section{Funding}

This present study was supported by the Hospital-level Topic of Maternal and Child Health Care Hospital of Shandong Province (grant no. 2020SFY001).

\section{Availability of data and materials}

The datasets used and/or analyzed during the current study are available from the corresponding author on reasonable request.

\section{Authors' contributions}

AL drafted the manuscript and performed the experiments. SL and $\mathrm{CZ}$ performed the experiments. ZF, YS and YP analyzed the data and organized the figures. YP revised the manuscript. $\mathrm{XW}$ and MZ designed the experiments and confirm the authenticity of all the raw data. All authors read and approved the final manuscript.

\section{Ethics approval and consent to participate}

This study was in accordance with the principles set out in the Declaration of Helsinki and its later amendments for ethical research involving human subjects. All procedures involving human participants were approved by the Ethics Committee of Maternal and child health care hospital of Shandong province (permit no: 2021-037). All patients signed informed consent for scientific research.

\section{Patient consent for publication}

Not applicable. 


\section{Competing interests}

The authors declare that they have no competing interests.

\section{References}

1. Joob B and Wiwanitkit V: Zika and spontaneous abortion. Bol Med Hosp Infant Mex 77: 46, 2020.

2. Van Leer P: Preventing spontaneous abortion with progestin therapy. Am Fam Physician 100: number 1, 2009.

3. Lee YH and Kim YC: Spontaneous resolution of serous retinal detachment caused by choroidal mass after a first trimester abortion. Yeungnam Univ J Med 37: 242-245, 2020.

4. Xiang H, Yan H, Sun B, Feng F and Chen P: Decreased expression of long non-coding RNA SNHG7 cause recurrent spontaneous abortion through suppression proliferation and invasion of trophoblast cells via miR-34a. Am J Transl Res 11: 463-472, 2019

5. Branch DW, Gibson M and Silver RM: Clinical practice. Recurrent miscarriage. N Engl J Med 363: 1740-1747, 2010.

6. Zong S, Li C, Luo C, Zhao X, Liu C, Wang K, Jia W, Bai M, Yin M, Bao S, et al: Dysregulated expression of IDO may cause unexplained recurrent spontaneous abortion through suppression of trophoblast cell proliferation and migration. Sci Rep 6: 19916, 2016.

7. Sun X, Tong X, Hao Y, Li C, Zhang Y, Pan Y, Dai Y, Liu L, Zhang T and Zhang S: Abnormal Cullin1 neddylation-mediated p21 accumulation participates in the pathogenesis of recurrent spontaneous abortion by regulating trophoblast cell proliferation and differentiation. Mol Hum Reprod 26: 327-339, 2020.

8. Li A, Zhang L, Li J, Fang Z, Li S, Peng Y, Zhang M and Wang X: Effect of RvD1/FPR2 on inflammatory response in chorioamnionitis. J Cell Mol Med 24: 13397-13407, 2020.

9. Murthi P, Rajaraman G, Erwich JJHM and Dimitriadis E: Decreased placental FPR2 in early pregnancies that later developed small-for-gestation age: a potential role of FPR2 in the regulation of epithelial-mesenchymal transition. Cells 9: E921, 2020.

10. Lappas M, McCracken S, McKelvey K, Lim R, James J, Roberts CT, Fournier T, Alfaidy N, Powell KL, Borg AJ, et al: Formyl peptide receptor- 2 is decreased in foetal growth restriction and contributes to placental dysfunction. Mol Hum Reprod 24: 94-109, 2018.

11. Xu Z, Zhao F, Lin F, Xiang H, Wang N, Ye D and Huang Y: Preeclampsia is associated with a deficiency of lipoxin A4, an endogenous anti-inflammatory mediator. Fertil Steril 102 : 282-290.e4, 2014.

12. Holdfeldt A, Sundqvist M, Dahlgren C and Forsman H: Data showing effects of a PI3K- $\delta$ inhibitor on neutrophil superoxide production during FPR2 activation and reactivation. Data Brief 32: 106185, 2020.

13. Liu GJ, Tao T, Wang H, Zhou Y, Gao X, Gao YY, Hang CH and Li W: Functions of resolvin D1-ALX/FPR2 receptor interaction in the hemoglobin-induced microglial inflammatory response and neuronal injury. J Neuroinflammation 17: 239, 2020.

14. Korimová A and Dubový P: N-Formylated peptide induces increased expression of both formyl peptide receptor 2 (Fpr2) and Toll-like receptor 9 (TLR9) in Schwannoma cells-an in vitro model for early inflammatory profiling of Schwann cells. Cells 9: E2661, 2020.

15. Hoxhaj G and Manning BD: The PI3K-AKT network at the interface of oncogenic signalling and cancer metabolism. Nat Rev Cancer 20: 74-88, 2020.

16. Nitulescu GM, Van De Venter M, Nitulescu G, Ungurianu A, Juzenas P, Peng Q, Olaru OT, Grădinaru D, Tsatsakis A, Tsoukalas D, et al: The Akt pathway in oncology therapy and beyond (Review). Int J Oncol 53: 2319-2331, 2018.

17. Livak KJ and Schmittgen TD: Analysis of relative gene expression data using real-time quantitative PCR and the 2- $\Delta \Delta C$ T method. Methods 25: 402-408, 2001.

18. Li Z, Zhou G, Jiang L, Xiang H and Cao Y: Effect of STOX1 on recurrent spontaneous abortion by regulating trophoblast cell proliferation and migration via the PI3K/AKT signaling pathway. J Cell Biochem 120: 8291-8299, 2018.

19. Cattaneo F, Russo R, Castaldo M, Chambery A, Zollo C, Esposito G, Pedone PV and Ammendola R: Phosphoproteomic analysis sheds light on intracellular signaling cascades triggered by Formyl-Peptide Receptor 2. Sci Rep 9: 17894, 2019.
20. Ammendola R,Parisi M,Esposito G and Cattaneo F: Pro-resolving FPR2 agonists regulate NADPH oxidase-dependent phosphorylation of HSP27, OSR1, and MARCKS and activation of the respective upstream kinases. Antioxidants (Basel) 10: 34, 2021.

21. Larqué E, Ruiz-Palacios M and Koletzko B: Placental regulation of fetal nutrient supply. Curr Opin Clin Nutr Metab Care 16: 292-297, 2013.

22. Waechter V, Schmid M, Herova M, Weber A, Günther V, Marti-Jaun J, Wüst S, Rösinger M, Gemperle C and Hersberger M: Characterization of the promoter and the transcriptional regulation of the lipoxin A4 receptor (FPR2/ALX) gene in human monocytes and macrophages. J Immunol 188: 1856-1867, 2012.

23. Lamouille S, Xu J and Derynck R: Molecular mechanisms of epithelial-mesenchymal transition. Nat Rev Mol Cell Biol 15: 178-196, 2014.

24. Burton GJ, Woods AW, Jauniaux E and Kingdom JC: Rheological and physiological consequences of conversion of the maternal spiral arteries for uteroplacental blood flow during human pregnancy. Placenta 30: 473-482, 2009.

25. Georgiades P, Ferguson-Smith AC and Burton GJ: Comparative developmental anatomy of the murine and human definitive placentae. Placenta 23: 3-19, 2002.

26. Silva JF and Serakides R: Intrauterine trophoblast migration: A comparative view of humans and rodents. Cell Adhes Migr 10: 88-110, 2016

27. Dagdelen M, Temur M, Yilmaz Ö, Altındag T, Uslu T and Özbay PO: Placental bed apoptosis is increased in pregnant women with pre-eclampsia versus normotensive pregnant women. J Obstet Gynaecol 36: 974-979, 2016.

28. Graham CH, Hawley TS, Hawley RG, MacDougall JR, Kerbel RS, Khoo N and Lala PK: Establishment and characterization of first trimester human trophoblast cells with extended lifespan. Exp Cell Res 206: 204-211, 1993.

29. Liu HN, Tang XM, Wang XQ, Gao J, Li N, Wang YY and Xia HF: miR-93 inhibits trophoblast cell proliferation and promotes cell apoptosis by targeting BCL2L2 in recurrent spontaneous abortion. Reprod Sci 27: 152-162, 2020.

30. Abou-Kheir W, Barrak J, Hadadeh O and Daoud G: HTR-8/SVneo cell line contains a mixed population of cells. Placenta 50: 1-7, 2017.

31. Li J, Tian J, Yin H, Peng Y, Liu S, Yao S and Zhang L: Chemical conjugation of FITC to track silica nanoparticles in vivo and in vitro: An emerging method to assess the reproductive toxicity of industrial nanomaterials. Environ Int 152: 106497, 2021.

32. Duan S, Zhang M, Li J, Tian J, Yin H, Wang X and Zhang L: Uterine metabolic disorder induced by silica nanoparticles: Biodistribution and bioactivity revealed by labeling with FITC. J Nanobiotechnology 19: 62, 2021.

33. Yin H, Li J, Tian J, Ma L, Zhang J, Zhai Q, Yao S and Zhang L: Uterine pyruvate metabolic disorder induced by silica nanoparticles act through the pentose phosphate pathway. J Hazard Mater 412: 125234, 2021

34. Yu Y, Xue S, Chen K, Le Y, Zhu R, Wang S, Liu S, Cheng X, Guan H, Wang JM, et al: The G-protein-coupled chemoattractant receptor Fpr2 exacerbates neuroglial dysfunction and angiogenesis in diabetic retinopathy. FASEB Bioadv 2: 613-623, 2020.

35. Matte A, Recchiuti A, Federti E, Koehl B, Mintz T, El Nemer W, Tharaux PL, Brousse V, Andolfo I, Lamolinara A, et al: Resolution of sickle cell disease-associated inflammation and tissue damage with 17R-resolvin D1. Blood 133: 252-265, 2019.

36. Rüger M, Kipp E, Schubert N, Schröder N, Pufe T, Stope MB, Kipp M, Blume C, Tauber SC and Brandenburg LO: The formyl peptide receptor agonist Ac2-26 alleviates neuroinflammation in a mouse model of pneumococcal meningitis. J Neuroinflammation 17: 325, 2020.

37. Vital SA, Senchenkova EY, Ansari J and Gavins FNE: Targeting AnxA1/Formyl Peptide Receptor 2 Pathway Affords Protection against Pathological Thrombo-Inflammation. Cells 9: E2473, 2020.

38. Xiang Y, Yao X, Chen K, Wang X, Zhou J, Gong W, Yoshimura T, Huang J, Wang R, Wu Y, et al: The G-protein coupled chemoattractant receptor FPR2 promotes malignant phenotype of human colon cancer cells. Am J Cancer Res 6: 2599-2610, 2016.

39. Lu J, Zhao J, Jia C, Zhou L, Cai Y, Ni J, Ma J, Zheng M and Lu A: FPR2 enhances colorectal cancer progression by promoting EMT process. Neoplasma 66: 785-791, 2019.

40. Sainz B Jr, Alcala S, Garcia E, Sanchez-Ripoll Y, Azevedo MM, Cioffi M, Tatari M, Miranda-Lorenzo I, Hidalgo M, Gomez-Lopez G, et al: Microenvironmental hCAP-18/LL-37 promotes pancreatic ductal adenocarcinoma by activating its cancer stem cell compartment. Gut 64: 1921-1935, 2015. 
41. Red-Horse K, Zhou Y, Genbacev O, Prakobphol A, Foulk R, McMaster $M$ and Fisher SJ: Trophoblast differentiation during embryo implantation and formation of the maternal-fetal interface. J Clin Invest 114: 744-754, 2004.

42. Zhao X, Jiang Y, Jiang T, Han X, Wang Y, Chen L and Feng X: Physiological and pathological regulation of autophagy in pregnancy. Arch Gynecol Obstet 302: 293-303, 2020.

43. Kawczak P, Bober L and Baczek T: Evaluation of Chemotherapeutic activity of the selected bases' analogues of nucleic acids supported by ab initio various quantum chemical calculations. Curr Computeraided Drug Des 16: 93-103, 2020.

44. Zhang W, Zuo M, Lu J and Wang Y: Adiponectin reduces embryonic loss rate and ameliorates trophoblast apoptosis in early pregnancy of mice with polycystic ovary syndrome by affecting the AMPK/PI3K/Akt/FoxO3a signaling pathway. Reprod Sci 27: 2232-2241, 2020.

45. Chen J, Yue C, Xu J, Zhan Y, Zhao H, Li Y and Ye Y: Downregulation of receptor tyrosine kinase-like orphan receptor 1 in preeclampsia placenta inhibits human trophoblast cell proliferation, migration, and invasion by PI3K/AKT/mTOR pathway accommodation. Placenta 82: 17-24, 2019.
46. Correia-Branco A, Keating E and Martel F: Involvement of mTOR, JNK and PI3K in the negative effect of ethanol and metformin on the human first-trimester extravillous trophoblast HTR-8/SVneo cell line. Eur J Pharmacol 833: 16-24, 2018.

47. Li Y, Sun XL, Ma CL, Li C, Zhan Y, Li WT, Li C and Wang YH: STX2 promotes trophoblast growth, migration, and invasion through activation of the PI3K-AKT pathway in preeclampsia. Front Cell Dev Biol 9: 615973, 2021.

48. Fresno Vara JA, Casado E, de Castro J, Cejas P, Belda-Iniesta C and González-Barón M: PI3K/Akt signalling pathway and cancer. Cancer Treat Rev 30: 193-204, 2004.

This work is licensed under a Creative Commons Attribution-NonCommercial-NoDerivatives 4.0 International (CC BY-NC-ND 4.0) License. 